\title{
湖南澧县大坪双堰东周水井出土古稻研究
}

\author{
向安强 ${ }^{1,2}$ 张文绪 $^{3}$ 曹 毅 ${ }^{4}$ 刘桂富 ${ }^{5}$
}

${ }^{1}$ 北京科技大学科技与文明研究中心, 北京 $100083 ;^{2}$ 华南农业大学公共管理学院社会学系, 广东广州 $510642 ;^{3}$ 中国农业大学农学与 生物技术学院, 北京 $100193 ;{ }^{4}$ 湖南省澧县博物馆, 湖南澧县 $415500 ;{ }^{5}$ 华南农业大学农学院, 广东广州 510642

摘 要: 对湖南澧县大坪双堰东周遗址水井出土的 3 粒炭化稻谷和 7 粒炭化稻米进行形态分析, 结果显示双堰古稻 具有大粒性和许多类似于粳型的形质特征(包括双峰乳突等), 表明它属于籼、粳之间的偏粳类型。与新石器时代遗址 出土的古稻谷相比, 双堰古稻的谷粒更宽、更厚和更重, 表明其谷粒有向短而圆的粳型变化的趋势。

关键词: 湖南澧县; 东周水井; 古稻; 形态特征; 双峰乳突; 亚种

\section{Ancient Rice Unearthed from Wells of the Eastern Zhou Dynasty Ruins at Daping Shuangyan of Li County in Hunan Province}

\author{
XIANG An-Qiang ${ }^{1,2}$, ZHANG Wen-Xu' ${ }^{3}, \mathrm{CAO} \mathrm{Yi}^{4}$, and LIU Gui-Fu ${ }^{5}$ \\ ${ }^{1}$ Research Centre for Science, Technology and Civilization, University of Science and Technology Beijing, Beijing 100083, China; ${ }^{2}$ Department of \\ Sociology, College of Public Management, South China Agricultural University, Guangzhou 510642, China; ${ }^{3}$ College of Agriculture and Biotechno- \\ logy, China Agricultural University, Beijing 100193, China; ${ }^{4}$ Li County Museum of Hunan Province, Li County 415500, China; ${ }^{5}$ College of Agricul- \\ ture, South China Agricultural University, Guangzhou 510642, China
}

\begin{abstract}
Three ancient carbonized unhusked rice and seven carbonized rice grains from wells of the Eastern Zhou Dynasty ruins at Daping Shuangyan in Hunan were morphologically analyzed. The results showed that Shuangyan ancient rice has the features of big grain and many similar morphological characteristics (including bi-peaked tubercles) to japonica rice, indicating that it belongs to the intermediate form between indica and japonica subspecies that is in the amesiality of japonica. Compared with the ancient unhusked rice unearthed from the Neolithic ruins, Shuangyan ancient rice has wider, thicker and heavier grains, tending to japonica characteristics of short and round grain.

Keywords: Li county in Hunan; Wells of the Eastern Zhou Dynasty ruins; Ancient rice; Morphological characteristics; Bi-peaked tubercles; Subspecies
\end{abstract}

水稻亚种分化是一个重要的课题, 不仅需要研 究史前的稻谷遗存, 历史时期的稻谷遗存研究同样 不可或缺。但是, 相对史前而言, 东周时期的稻作考 古, 无论是发现还是研究都显得十分有限。东周时 期的稻作农业较史前有了很大发展, 栽培稻种进化, 种植面积扩大, 稻谷产量增加; 但不知何故, 东周 时期的稻作考古发现却很少或未见报道，东周古稻 的分析研究更是几乎为零。从栽培稻演化的发展序 列来看, 东周栽培稻处于中间环节, 可能是介于古 栽培稻与现代稻种之间的种群; 可谓承前启后, 非 常重要。双堰水井古稻属于东周时期, 是演化相对
居于中期并趋于成熟的栽培水稻; 研究东周水井中 出土的谷、米等稻谷遗存粒型以及双峰乳突形态, 对我们思考稻作农业起源和籼粳分化等问题具有参 考意义; 观察分析其特征, 有利于进一步认识和研 究中国栽培水稻的演化方向。

\section{1 材料与方法}

\section{1 遗址概况与材料}

双堰遗址位于湖南省常德市澧县大坪镇群乐 村。1989 年因砖厂生产取土遭破坏而对其进行了抢 救性发掘。采集和发掘到的陶器(片)的代表器物有

本研究由广东省自然科学基金项目(10151064201000059)资助。 第一作者联系方式: E-mail: xianganqiang@126.com Received(收稿日期): 2012-03-31; Accepted(接受日期): 2012-06-30; Published online(网络出版日期): 2012-07-27. URL: http://www.cnki.net/kcms/detail/11.1809.S.20120727.0846.018.html 
豆、鬲、针等，属于东周时期的楚文化。在 $8000 \mathrm{~m}^{2}$ 的范围内发现了 6 眼可能用于农田灌溉的水井, 井 与井之间的最大间距为 $60 \mathrm{~m}$, 最小间距为 $20 \mathrm{~m}$; 对 其中破坏严重的 4 眼水井进行了挖掘清理, 通过对 井中填土的篮选和漂洗, 获得了一批稻谷、桃、杏、 瓜等农作物遗存; 在 2 号和 4 号井中发现了大量烧 过的稻草灰, 其厚度近 $1 \mathrm{~m}$, 内含大量形态完整的 稻谷壳和少量形态尚完整的稻谷(米)颗粒 ${ }^{[1]}$ 。本研究 对出土稻谷和稻米进行了观察分析。

材料由湖南澧县博物馆提供炭化稻 3 粒, 炭化 米 7 粒, 以及只有完整的炭化稻稃而无米粒的材料 20 粒。

参照系由中国农业大学提供, 有普通野生稻、 籼稻、粳稻和八十垱古稻 ${ }^{[2-4]}$ 。

\section{2 方法}

对 7 粒炭化米和 3 粒炭化稻进行编号, 在体视 镜下用测微尺逐粒测量长、宽、厚。

粒长乘以 1.5225 , 粒宽乘以 1.3004 , 粒厚乘以 1.1858 系数, 将其转化为新鲜稻谷; 炭化稻谷则将 粒长加 $7.67 \%$ 收缩系数, 以便在相同条件下与参照 系进行比较研究 ${ }^{[5]}$ 。

两性状平均数差异显著性测定用以下公式进行:

$$
D_{\bar{x}_{1}-\bar{x}_{2}}=\frac{\bar{x}_{1}-\bar{x}_{2}}{\sqrt{\frac{\sigma_{1}^{2}}{n_{1}}+\frac{\sigma_{2}^{2}}{n_{2}}}}
$$

以符号 ns 表示差异不显著; 该值 $>2$, 为差异显 著, 以符号 $*$ 表示; 该值 $>3$, 为差异极显著, 以符号 **表示。式中 $\bar{x}_{1}-\bar{x}_{2}$ 为 2 个性状平均数差值; $\sigma_{1}{ }^{2}$ 和 $\sigma_{2}{ }^{2}$ 分别为两性状测定值的方差; $n_{1}$ 和 $n_{2}$ 分别为 2 个 性状的测定次数。

稻种间形质的距离测定, 先将数据总体标准化, 然后按欧氏距离公式进行测定。

$$
E D=\sqrt{\sum\left(x_{i j}-x_{i k}\right)^{2}}
$$

式中 $i$ 为性状, $j$ 和 $k$ 为种群。

粒型的属性判别 ${ }^{[6]}$ ，依以下函数公式计算。

$$
\begin{aligned}
& f_{1}=175.3+48.8362 x_{1}-3.9591 x_{2} \\
& f_{2}=-131.086+39.816 x_{1}-2.4795 x_{2} \\
& f_{3}=-96.5161+32.2803 x_{1}-1.5615 x_{2}
\end{aligned}
$$

式中, $f_{1} 、 f_{2} 、 f_{3}$ 分别为野、籼、粳的判别函数, 粒 型属性属于三者中的数值最大者; $x_{1}$ 和 $x_{2}$ 分别为谷 长和谷重, 谷重 $=3.6238 \times$ (谷长 $\times$ 谷宽 $\times$ 谷厚 $) / 8$ 。

双峰乳突判别函数 ${ }^{[7-8]}$ 为 BPT.G $(x)=44.4788-$ $0.4308 \times B P D-0.4263 \times P A+0.075 \times B P D / C D, \quad B P T . G(x)$
负值表示粳型, 正值表示籼型。式中 $B P D$ 为双峰距; $P A$ 为峰角度; $B P D / C D$ 为双峰距和垭深的比值。

\section{2 结果与分析}

\section{1 双堰东周遗址古稻特征}

如图 1 和表 1 所示, 双堰东周古稻的谷长 $8.65 \mathrm{~mm}$, 比参照系中最长的普通野生稻 $(8.56 \mathrm{~mm})$ 长 $0.09 \mathrm{~mm}$, 可认为二者基本相同; 谷宽 $3.62 \mathrm{~mm}$, 则比参照系中 最宽的粳稻 $(3.34 \mathrm{~mm})$ 宽 $0.28 \mathrm{~mm}$; 谷厚为 $2.41 \mathrm{~mm}$, 比最厚的粳稻 $(2.28 \mathrm{~mm})$ 厚 $0.13 \mathrm{~mm}$; 估测谷重 35.27 $\mathrm{mg}$, 是一种又大又重的种群。其长/宽比 2.41 , 处于籼、 粳之间，显示出一种籼粳中间类型状态。



图 1 双堰古稻及其参照系

Fig. 1 Grain figures of Shuangyan ancient rice and the reference rice

\section{2 双堰古稻与参照系性状差异显著性测定} 如表 2 所示, 双堰古稻与 4 个参照系的谷长、 谷宽、谷厚、长宽比、谷重等性状比校，除了与普 通野生稻和籼稻在谷粒长度方面没有显著差异外, 其他的差异都显著或极显著。

古稻与普通野生稻和籼稻的谷长没有显著差异, 而与粳稻差异极显著, 是因为古稻谷长较长所致。 而在谷宽、谷厚和谷重性状上，与普通野稻、八十 垱古稻和籼稻差异极显著, 与粳稻却只达显著差异 水平，表明古稻性状趋向宽、厚、重，即大粒型。 粒型的长/宽比与普通野稻、八十垱古稻和籼稻差异 极显著, 而与粳稻差异显著, 表明古稻粒型有向短 而圆的倾向。

\section{3 双堰古稻群中的粒型分析}

用粒型判别函数对双堰古稻逐粒判别, 结果有 1 粒为类野稻粒型, 5 粒为类籼稻粒型, 4 粒为类粳稻 粒型。 
表 1 双堰东周古稻的粒形特征

Table 1 Grain shapes of Shuangyan ancient rice unearthed at the Eastern Zhou Dynasty ruins

\begin{tabular}{|c|c|c|c|c|c|c|}
\hline $\begin{array}{c}\text { 稻类 } \\
\text { Rice type }\end{array}$ & $\begin{array}{l}\text { 项目 } \\
\text { Item }\end{array}$ & $\begin{array}{c}\text { 谷长 } \\
\text { Grain length } \\
(\mathrm{mm})\end{array}$ & $\begin{array}{c}\text { 谷宽 } \\
\text { Grain width } \\
\text { (mm) }\end{array}$ & $\begin{array}{c}\text { 谷厚 } \\
\text { Grain thick- } \\
\text { ness (mm) }\end{array}$ & $\begin{array}{c}\text { 谷长/谷宽 } \\
\text { Grain length/ } \\
\text { grain width }\end{array}$ & $\begin{array}{c}\text { 谷重 } \\
\text { Grain } \\
\text { weight (mg) }\end{array}$ \\
\hline 双堰古稻 & 平均值 $\mathrm{Mean} \pm S D$ & $8.65 \pm 0.99$ & $3.62 \pm 0.43$ & $2.41 \pm 0.51$ & $2.41 \pm 0.31$ & $35.27 \pm 13.26$ \\
\hline Shuangyan ancient rice & 变异系数 CV (\%) & 11.50 & 11.87 & 21.10 & 12.81 & 37.58 \\
\hline 普通野生稻 & 平均值 Mean $\pm S D$ & $8.58 \pm 0.54$ & $2.58 \pm 0.30$ & $1.79 \pm 0.18$ & $3.53 \pm 0.38$ & $18.16 \pm 4.07$ \\
\hline Oryza rufipogon & 变异系数 CV (\%) & 6.30 & 12.00 & 9.80 & 11.00 & 22.00 \\
\hline 八十垱古稻 & 平均值 Mean $\pm S D$ & $7.91 \pm 0.74$ & $2.88 \pm 0.32$ & $1.98 \pm 0.30$ & $2.77 \pm 0.33$ & $20.65 \pm 5.34$ \\
\hline Eighty dang ancient rice & 变异系数 CV (\%) & 9.00 & 11.00 & 15.00 & 12.00 & 26.00 \\
\hline 籼稻 & 平均值 Mean $\pm S D$ & $8.02 \pm 0.51$ & $2.94 \pm 0.24$ & $2.09 \pm 0.12$ & $2.75 \pm 0.30$ & $22.30 \pm 2.97$ \\
\hline Indica & 变异系数 CV (\%) & 6.30 & 8.30 & 5.90 & 10.80 & 13.30 \\
\hline 粳稻 & 平均值 Mean $\pm S D$ & $7.43 \pm 0.58$ & $3.34 \pm 0.26$ & $2.28 \pm 0.15$ & $2.20 \pm 0.20$ & $24.75 \pm 4.14$ \\
\hline Japonica & 变异系数 CV (\%) & 7.80 & 7.70 & 6.70 & 8.90 & 16.70 \\
\hline
\end{tabular}

表 2 双堰古稻与参照系谷粒性状差异显著性

Table 2 Grain differences between Shuangyan ancient rice and the reference rice

\begin{tabular}{|c|c|c|c|c|c|}
\hline $\begin{array}{c}\text { 参照系 } \\
\text { Reference rice }\end{array}$ & $\begin{array}{c}\text { 谷长 } \\
\text { Grain length } \\
(\mathrm{mm})\end{array}$ & $\begin{array}{c}\text { 谷宽 } \\
\text { Grain width } \\
(\mathrm{mm})\end{array}$ & $\begin{array}{c}\text { 谷厚 } \\
\text { Grain thickness } \\
(\mathrm{mm})\end{array}$ & $\begin{array}{c}\text { 谷长/谷宽 } \\
\text { Grain length/ } \\
\text { grain width }\end{array}$ & $\begin{array}{c}\text { 谷重 } \\
\text { Grain weight } \\
\text { (mg) }\end{array}$ \\
\hline 普通野生稻 Oryza rufipogon & $0.22^{\mathrm{ns}}$ & $7.55^{* *}$ & $10.6^{* *}$ & $9.22^{* *}$ & $4.07^{* *}$ \\
\hline 八十垱古稻 Eighty dang ancient rice & $2.35^{*}$ & $3.04^{* *}$ & $7.44^{* *}$ & $3.65^{* *}$ & $3.48^{* *}$ \\
\hline 籼稻 Indica & $1.99^{\mathrm{ns}}$ & $11.45^{* *}$ & $5.56^{* *}$ & $3.99^{* *}$ & $3.09^{* *}$ \\
\hline 粳稻 Japonica & $3.86^{* *}$ & $2.04^{*}$ & $2.24^{*}$ & $2.12^{*}$ & $2.50^{*}$ \\
\hline
\end{tabular}

ns ${ }^{*},{ }^{* *}$ 分别表示不显著、0.05 和 0.01 水平的显著。

ns , ${ }^{*}$, and ${ }^{* *}$ indicate the no significance, the significance at 0.05 and 0.01 levels, respectively.

双堰古稻群体平均值判别, 类似粳稻的函数值 为 127.71 , 类似籼稻的函数值为 125.96 , 类似普通 野稻的函数值仅为 107.61 。可以看到, 类似粳稻和 籼稻的函数值非常接近, 而离普通野稻较远。就群 体判别可认为倾向粳型, 但属于籼、粳中间状态的 倾粳类型。

\section{4 双堰古稻与参照系的形质欧氏距离分析}

通过距离测定，双堰古稻与普通野生稻的距离 为 18.1 , 与八十垱古稻距离为 14.0 , 与籼稻距离为 12.3 ，与粳稻距离为 9.6 (表 3)。这个结果表明该群 古稻的各项形质特征更相近于粳稻, 与粒型判别结 果一致。

表 3 双堰古稻与参照系表型的欧氏距离

Table 3 Euclidean distances between Shuangyan ancient rice and the reference rice

\begin{tabular}{lc}
\hline \multicolumn{1}{c}{ 参照系 } & $\begin{array}{c}\text { 欧氏距离 } \\
\text { Reference rice }\end{array}$ \\
\hline 普通野稻 Oryza rufipogon & 18.1 \\
八十垱古稻 Eighty dang ancient rice & 14.0 \\
籼稻 Indica & 12.3 \\
粳稻 Japonica & 9.6 \\
\hline
\end{tabular}

\section{5 双堰古稻的双峰乳突特征}

粳稻、籼稻和普通野生稻 3 类参照系稻种的双 峰乳突形态显著不同(图 2), 可用判别函数加以区 分 ${ }^{[7-8]}$ 。如表 4 所示, 双堰古稻双峰乳突特征属粳型。

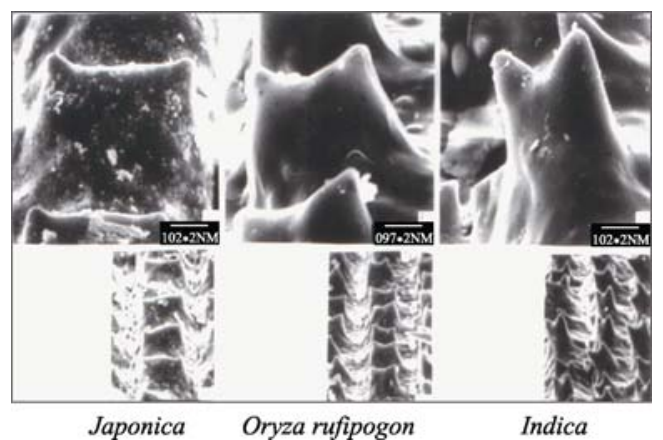

图 2 现代 3 类稻种的双峰乳突特征

Fig. 2 Features of bi-peaked tubercles for three types of modern rices

双堰古稻双峰距 $36.67 \mu \mathrm{m}$, 稍大于粳稻的 33.25 $\mu \mathrm{m}$ ，大大超过了其余 3 种稻类，属于宽大的双峰乳 突类型。垭深 $4.50 \mu \mathrm{m}$, 接近普通野生稻的 $4.79 \mu \mathrm{m}$, 从垭深特征可看到古稻演化过程留下的痕迹; 古稻 的峰角度 87.28 , 接近粳稻的 88.82 , 明显大于其余 3 
种稻类; 判别函数值为 -7.62 , 属于稳态的粳型双峰 乳突(图 3)。

表 4 各类稻双峰乳突的特征

Table 4 Features of bi-peaked tubercles for various types of rice

\begin{tabular}{|c|c|c|c|c|c|c|c|}
\hline $\begin{array}{c}\text { 稻类 } \\
\text { Rice type }\end{array}$ & $\begin{array}{l}\text { 样本数 } \\
\text { Sample } \\
\text { size }(n) \\
\end{array}$ & $\begin{array}{l}\text { 项目 } \\
\text { Item }\end{array}$ & $\begin{array}{c}\text { 双峰距 } \\
\text { Distance of } \\
\text { bi-peaks }(\mu \mathrm{m})\end{array}$ & $\begin{array}{c}\text { 垭深 } \\
\text { Col deep } \\
(\mu \mathrm{m}) \\
\end{array}$ & $\begin{array}{c}\text { 距深比 } \\
\text { Distance of } \\
\text { bi-peaks / col deep }\end{array}$ & $\begin{array}{c}\text { 峰角 } \\
\text { Angle of } \\
\text { peak }\left(^{\circ}\right)\end{array}$ & $\begin{array}{c}\text { 判别函数值 } \\
\text { Discrimination } \\
\text { function }\end{array}$ \\
\hline \multirow{2}{*}{$\begin{array}{l}\text { 双堰古稻 } \\
\text { Shuangyan ancient rice }\end{array}$} & \multirow{2}{*}{40} & 平均值 Mean $\pm S D$ & $36.67 \pm 7.31$ & $4.50 \pm 2.02$ & $12.09 \pm 13.73$ & $87.28 \pm 6.93$ & \multirow{2}{*}{-7.62} \\
\hline & & 变异系数 CV (\%) & 19.93 & 44.81 & 113.56 & 7.94 & \\
\hline 普通野稻 & \multirow{2}{*}{89} & 平均值 Mean $\pm S D$ & $27.69 \pm 5.23$ & $4.79 \pm 1.07$ & $6.02 \pm 1.72$ & $71.56 \pm 7.00$ & \multirow{2}{*}{2.50} \\
\hline Oryza rufipogon & & 变异系数 CV (\%) & 18.87 & 22.22 & 28.55 & 9.79 & \\
\hline 八十垱古稻 & \multirow{2}{*}{37} & 平均值 Mean $\pm S D$ & $25.65 \pm 4.66$ & $3.28 \pm 0.93$ & $8.66 \pm 3.70$ & $72.52 \pm 11.24$ & \multirow{2}{*}{3.16} \\
\hline Eighty dang ancient rice & & 变异系数 CV (\%) & 18.18 & 28.25 & 42.79 & 15.49 & \\
\hline 籼稻 & \multirow{2}{*}{70} & 平均值 Mean $\pm S D$ & $23.23 \pm 3.96$ & $4.38 \pm 1.09$ & $5.61 \pm 1.63$ & $67.83 \pm 6.96$ & \multirow{2}{*}{5.94} \\
\hline Indica & & 变异系数 CV (\%) & 17.06 & 24.89 & 28.98 & 10.26 & \\
\hline 粳稻 & \multirow{2}{*}{80} & 平均值 Mean $\pm S D$ & $33.25 \pm 5.19$ & $1.84 \pm 1.09$ & $27.29 \pm 23.83$ & $88.82 \pm 8.97$ & \multirow{2}{*}{-5.48} \\
\hline Japonica & & 变异系数 CV (\%) & 15.61 & 59.33 & 87.31 & 10.10 & \\
\hline
\end{tabular}

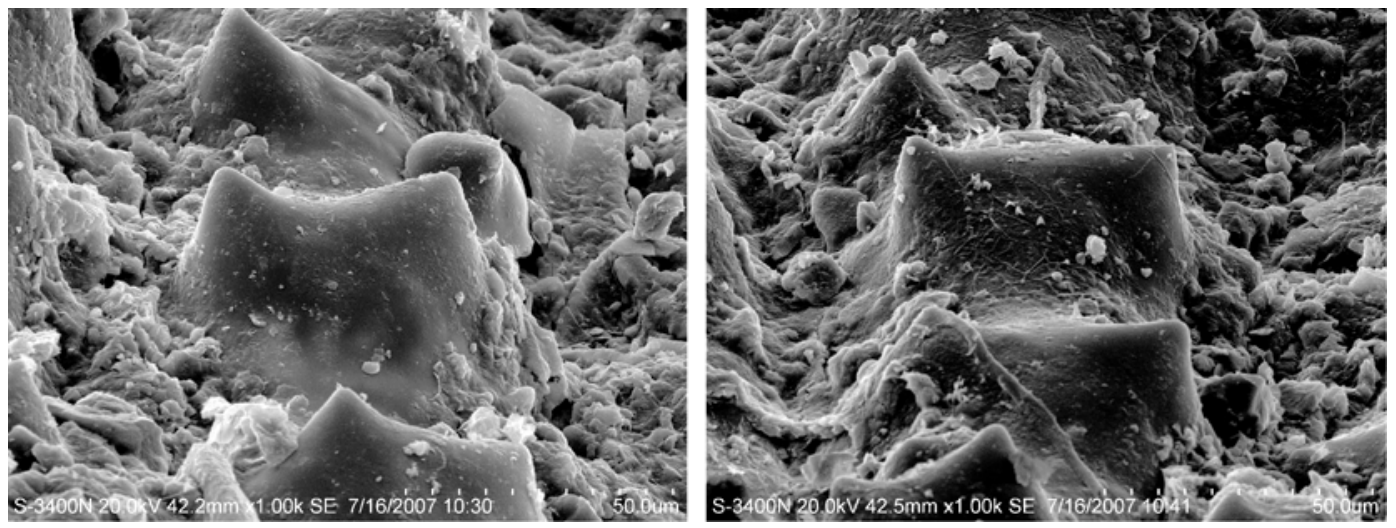

图 3 双堰古稻的双峰乳突

Fig. 3 Features of bi-peaked tubercles for Shuangyan ancient rice

\section{3 讨论与结论}

东周已经进入青铜器文化时期, 科技水平远高 于新石器时代。农业生产方面人们根据需要对栽培 水稻的干预亦逐渐增多, 人工选择的强度必渐加大, 因此对稻种特征的演化方向必然产生影响。

双堰古稻的出土地点位于澧阳平原, 濒临洞庭 湖滨, 是古稻栽培极发达、历史极久远的地区, 直到 现在仍是水稻主产重地。澧阳平原稻作文化开始于 万年前, 迄今水稻栽培一脉相承。从距今 9000 年前 的彭头山古稻 ${ }^{[9]}$ 、八十垱古稻 ${ }^{[2-4]}$ 和距今 6000 年前 的城头山古稻 ${ }^{[10]}$, 到距今 3000 年前的双堰东周古 稻, 再发展到当今澧县平原的现代水稻; 其东周古 稻和西汉古稻, 则是稻作历史的重要中间环节。城 头山古稻已具有粒大粒重的特征, 其后的双堰东周 古稻的粒大粒重性特征更趋明显; 二者的关系值得
研究, 有待学术界深入探讨。所以, 双堰东周古稻及 分析材料, 对研究栽培水稻的演化历史和特征极其 珍贵，值得学术界重视。

该群古稻具有 2 个鲜明特征, 大粒性和倾粳性, 这与人们的食味兴趣、加工需求和对产量的提高不 无关系。

\section{References}

[1] Xiang A-Q(向安强). Agricultural remains from the Eastern Zhou Dynasty ruins in Li County of Hunan. Agric Archaeol (农业考 古), 1992, (1): 123-127 (in Chinese)

[2] Zhang W-X(张文绪), Pei A-P(裴安平). Bashidang ancient rice unearthed at Mengxi in Li country. Cult Realic (文物), 1997, (1): 36-41 (in Chinese)

[3] Zhao D-L(赵笃乐), Pei A-P(裴安平), Zhang W-X(张文绪). Restudy of ancient cultivated rice from Bashidang site in Lixian 
country, Hunan province. Chin J Rice Sci (中国水稻科学), 2000, 14(3): 139-143 (in Chinese with English abstract)

[4] Zhang W-X(张文绪), Pei A-P(裴安平). The study of grain multitypes of ancient cultivated rice of Bashidang ruins in Lixian county of Hunan province. Acta Agron Sin (作物学报), 2002, 28(1): 90-93 (in Chinese with English abstract)

[5] Zhang W-X(张文绪), Pei A-P(裴安平). The study of the ancient cultivated rice restored from the carbonized rice. Acta Agron Sin (作物学报), 2000, 26(5): 579-586 (in Chinese with English abstract)

[6] Zhang W-X(张文绪), Pei X-D(裴金德). Study of ancient cultivated rice by using characters of rice grain. J China Agric Univ (中国农业大学学报), 2000, 5(3): 41-44 (in Chinese with English abstract)

[7] Zhang W-X(张文绪). Observation of bi-peaked tubercles at the lemma in rice by using scanning electron microscope. Beijing Agric Univ J (北京农业大学学报), 1995, 21(2): 143-146 (in Chinese)

[8] Zhang W-X(张文绪), Pei X-D(裴鍂德). Studies on the bipeaked tubercles of Lemma in rice. Acta Agron Sin (作物学报), 1998, 24(6): 691-697 (in Chinese with English abstract)

[9] Zhang W-X(张文绪), Pei A-P(裴安平), Mao T-L(毛同林). Studies on the printings of bi-peaked tubercles on lemmas of rice kernels in broken pottery pieces unearthed from pengtoushan excavation site in Li County, Hunan. Acta Agron Sin (作物学报), 2003, 29(2): 263-267 (in Chinese with English abstract)

[10] Zhang W-X(张文绪), Gu H-B(顾海滨). Study on the ancient rice at Chengtoushan ruins in Lixian county, Hunan province. Acta Agron Sin (作物学报), 2005, 31(6): 737-741 (in Chinese with English abstract)

\section{科学出版社生物分社新书推介}

\section{《实用生物统计学》}

编著者：顾志峰，叶乃好，石耀华

出版时间: 2012 年 8 月

书号: 9787030347626

定价: 58 元

《实用生物统计学》主要针对生命科学研究领域中的常见问题, 以生 物统计学的基础理论知识、试验资料的收集整理、资料的统计分析和试验 设计为主线来安排主要内容。每章内容先以具体的科学问题引出相应的生 物统计学问题, 然后对其基本理论进行介绍, 结合相应的统计软件(包括 SPSS、DPS、Minitab、Excel 等)对实际问题进行具体分析, 同时采用截图 法对具体使用的统计方法进行直观形象的介绍, 最后结合专业知识对分析

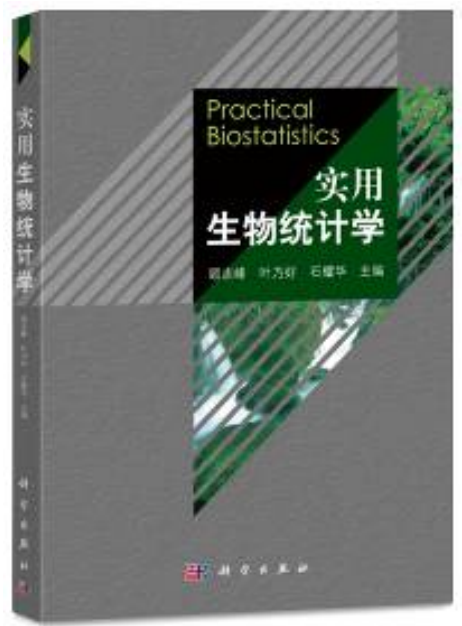
结果进行科学阐释。《实用生物统计学》的设计和编排使每位读者能够很清 晰地理解每个问题的解题要领, 科学合理地选用相应的统计软件来分析和解决实际问题, 同时能够很直观、 形象地掌握每种软件的使用方法和技巧。

购书指南 订购方式 1——网上购书: 淘宝商城科学出版社旗舰店 http://kxcbs.tmall.com/; 卓越亚马逊 http://www.amazon.cn/; 当当网 http://www.dangdang.com/; 京东图书 http://book.360buy.com/

订购方式 2-一电话购书, 联系人: 科学出版社 贾海涛, 电话 13501022258, 010-64017321

订购方式 3——邮件购书: 生物分社: lifescience@mail.sciencep.com; 贾海涛: jiahaitao@mail.sciencep.com 